\title{
Understanding the effects of dietary components on the gut microbiome and human health
}

\author{
Bryna Rackerby $^{1} \cdot$ Hyun Jung Kim ${ }^{2}$ David C. Dallas ${ }^{3} \cdot$ Si Hong Park ${ }^{1}$ (D)
}

Received: 15 June 2020/Revised: 4 August 2020/Accepted: 18 August 2020

(C) The Author(s) 2020, corrected publication 2020

\begin{abstract}
The gut microbiome is the complex microbial ecosystem found in the gastrointestinal tract of humans and animals. It plays a vital role in host development, physiology and metabolism, and has been implicated as a factor in brain function, behavior, mental health, and many disease states. While many factors, including host genetics and environmental factors, contribute to the composition of the gut microbiome, diet plays a large role. Microorganisms differ in their nutrient requirements, and alterations in host dietary composition can have strong impacts on the microbial inhabitants of the gastrointestinal tract. The health implications of these dietary and microbial changes are relevant as various global populations consume diets comprised of different macronutrient ratios, and many diets promote alterations to recommended macronutrient ratios to promote health. This review will outline the ways in which specific macro- and micronutrients impact the gut microbiome and host health.
\end{abstract}

Si Hong Park

sihong.park@oregonstate.edu

Bryna Rackerby

bryna.rackerby@oregonstate.edu

Hyun Jung Kim

hjkim@kfri.re.kr

David C. Dallas

dave.dallas@oregonstate.edu

1 Department of Food Science and Technology, Oregon State University, Corvallis, OR 97331, USA

2 Korea Food Research Institute, Wanju, Jeollabuk-do 55365, South Korea

3 School of Biological and Population Health Sciences, Nutrition, Oregon State University, Corvallis, OR 97331, USA
Keywords Diet - Gut microbiome - Macronutrient . Micronutrient · Health

\section{Introduction}

The gut microbiome is the microbial ecosystem comprised of the many different organisms residing in the gastrointestinal tract. Two phyla, Bacteroidetes and Firmicutes, dominate the intestinal environment (Eckburg et al., 2005). Though the gut microbiome is highly dynamic, evidence suggests that we can only alter 30 to $40 \%$ of our adult gastrointestinal microbiome; the other 60 to $70 \%$ is likely controlled by factors such as genetics, epigenetics, and maternal factors such as maternal health, diet, and breastfeeding (Kashtanova et al., 2016). Many of the over 5 million collective genes of the gut microbiome encode biosynthetic enzymes, glycosidases, and proteases (Sommer and Bäckhed, 2013). The gut microbiome contributes to a wide range of functions vital to the host including immune regulation, organ development, host metabolism, behavior (Sommer and Bäckhed, 2013), and maintenance of the structural integrity of the intestinal mucosa (Jandhyala et al. 2015). A dysbiotic gut microbiome can contribute to health problems and is often characterized by reduced diversity, increased pathobionts, or microbial overgrowth. The intestinal microbiota is thought to play a role not only in inflammatory conditions associated with diet such as inflammatory bowel disease (IBD), obesity, diabetes (Clarke et al., 2014), and cardiovascular disease (Sandoval and Seeley, 2010), but also in other health-related conditions like asthma (Clarke et al., 2014), autism (Vuong and Hsiao, 2017), epilepsy (Dahlin and PrastNielsen, 2019), depression, and schizophrenia (Robertson et al., 2017). Research indicates that the gut microbiome is 
a contributing factor in certain phenotypic traits and conditions (O'Hara and Shanahan, 2006).

Healthy microbiomes can influence the growth of undesirable organisms in a range of environments, intestinal and otherwise. For example, the wooden vats used for making Protected Designation of Origin (PDO) Salers cheese are not sterilized and contain biofilms that are protective against pathogens. Even when milk is inoculated with Listeria monocytogenes or Staphylococcus aureus, these organisms are found neither on the wooden surfaces nor in the resulting cheeses (Didienne et al., 2012). This example and many others demonstrate that promoting the growth of beneficial organisms is an effective method to prevent the growth of pathogens. These ideas have been implemented in mainstream therapies and in food safety (Niederwerder, 2018). For example, fecal transplants on young chicks are effective in reducing colonization by pathogens like Salmonella in poultry by establishing Salmonella-free intestinal microbiomes (Snoeyenbos et al., 1982). Not only does early institution of a healthy microbiome prevent overgrowth of pathobionts already present in the gut as well as pathogenic infections in chickens, a healthy gut microbiome can help prevent dysbiosis and pathogenic infection in humans (Kamada et al., 2013). A dysbiotic gut environment is less resistant to infection as pathogens can readily outcompete commensal organisms due to their resistance against host defenses. A more diverse diet results in a more diverse intestinal microbiome (Heiman and Greenway, 2016), which can help prevent certain dysbioses that are exploited by intestinal pathogens and lead to infection. In addition to protecting against infection or overgrowth, a healthy gut microbiota also acts as a barrier that modulates host interactions with xenobiotics (Gaulke et al., 2018).

Dietary components can be divided into three macronutrients-carbohydrates, proteins, and fats, and two types of micronutrients-vitamins and minerals. Macronutrients are required for energy production and anabolism while micronutrients are typically enzymatic cofactors in biochemical reactions. Microbes require nutrients just as humans and animals do, however they are unique in that they both use and produce vitamins. Therefore, microorganisms have a complex relationship with intestinal vitamin levels. Dietary macronutrient ratios and food preparation methods can also influence the gut microbiome and impact the metabolic profile of the gut microbiota (Maier et al., 2017; Shen et al., 2010). The traditional diets of cultures across the world vary in macronutrient ratios. Further, many alternative diets promote altered macronutrient ratios as a means to lose weight or promote health. Therefore, the impact of dietary macroand micronutrient composition on the intestinal microbiota is highly relevant.

\section{Macronutrients}

\section{Carbohydrates}

The capacity of the intestinal microbiota to metabolize carbohydrates is potentially several thousand times our own genome-encoded ability (Sonnenburg and Sonnenburg, 2014). Carbohydrates can be classified as mono-, di-, oligo-, or polysaccharides. Because only monosaccharides can be taken up through the intestinal wall, larger molecules must first be broken down into their basic subunits prior to absorption (Huber et al., 2017). Sugar is rapidly absorbed in the small intestine where microbial genes related to carbohydrate metabolism are overrepresented compared to the distal colon (Zoetendal et al., 2012). Carbohydrates can be divided into three basic groups that are of nutritional importance: starch, fiber, and simple sugar. Starch is produced by plants as their primary food reserve. Starches are the only human-digestible polysaccharides, as humans lack the glycosidases necessary to cleave the $\beta-1,4$ glycosidic bonds found in other polysaccharides. Fiber consists of non-digestible oligo- and polysaccharides, as well as some forms of starch referred to as resistant starch (Huber et al., 2017).

\section{Fiber}

Fiber can be grouped into soluble and insoluble fibers, with soluble fibers conferring health benefits like increased intestinal and fecal bulk, decreased transit time, and decreased serum cholesterol (Huber et al., 2017). As fiber is undigestible by the human gastrointestinal tract, it remains relatively intact as it enters the colon where it is accessible to the intestinal microbiota. A low fiber diet not only affects the gut microbiota of the individual consuming it, but can also reduce the microbial diversity of offspring in a way that is irreversible by fiber alone over only a few generations (Sonnenburg et al., 2016). Whole grains are an example of a high fiber food that contains a variety of microbially accessible compounds, such as beta-glucans and arabinoxylans (Gong et al., 2018). Consumption of whole-grain products can increase Bifidobacterium, Lactobacillus, Enterococcus, Blautia, and Roseburia, increase the Firmicutes:Bacteroidetes ratio, and decrease Bacteroides (Graf et al., 2015). Thirty-nine out of 42 studies also found that cereal fibers increased microbial diversity, abundance, or both (Jefferson and Adolphus, 2019).

Whole grain oat flour increased levels of Alcaligenaceae, which can metabolize oestradiol in vitro and may promote the reabsorption of cholesterol (Vitaglione et al., 2019). Some research indicates that most of the impact of grains on the gut microbiome is attributable to phenolic 
compounds, beta-glucans, and arabinoxylans (Gong et al., 2018), though one paper reported that neither beta-glucans nor polyphenols were responsible for the bifidogenic effect associated with oat consumption (Kristek et al., 2019). Different beta-glucans have various effects on the gut microbiome. Low molecular weight beta-glucans did not alter the microbiomes of human subjects while high molecular weight beta-glucans decreased the Firmicutes:Bacteroidetes ratio, with increases in Bacteroides and Prevotella, and decreases in Dorea (Wang et al., 2016). The model organism influences the observed microbial impact as well. Oat beta-glucan increased Lactobacillus and Bifidobacterium and decreased Enterobacteriaceae in rats (Shen et al., 2012), whereas in pigs it increased Lactobacillus, Streptococcus, Enterococcus, Clostridium clusters I and XIVa, certain members of BacteroidesPrevotella-Porphyromonas, and Enterobacteriaceae (Metzler-Zebeli et al., 2011).

Arabinoxylans are the most abundant non-starch polysaccharides present in cereal grains and contribute significantly to gut health (Mendis et al., 2016). Arabinoxylans have been associated with increased Bifidobacterium (specifically Bifidobacterium animalis ssp. lactis), Prevotella, Faecalibacterium prausnitzii, and Lactobacillus as well as decreased Escherichia coli, Streptococcus, Staphylococcus, Lactobacillus, Clostridium histolyticum I and II, and Enterococcus (Gong et al., 2018). Long-chain arabinoxylans also promote the growth of Bifidobacterium longum with a concurrent increase in propionate levels (Van den Abbeele et al., 2013) and have been associated with immunomodulatory properties in a variety of organisms (Mendis et al., 2016).

While most starch is digestible, resistant starch (RS) is non-digestible and is therefore classified as a fiber. Resistant starch is traditionally categorized into four groups: RS1 is physically inaccessible, RS2 is ungelatinized raw starch, R3 is retrograded starch, and RS4 is chemically modified resistant starch (Kashantova et al., 2016). Eubacterium rectale, Ruminococcus bromii, and Bifidobacterium thetaiotamicron and adolescentis are important organisms in the degradation of RS (Ze et al., 2012). Ruminococcus bromii and B. adolescentis are better able to ferment RS2 and RS3 than E. rectale or B. thetaiotamicron, however in co-culture $R$. bromii stimulated the ability of the other three organisms to degrade these compounds. Further, addition of $R$. bromii to fecal microbiomes devoid of $R$. bromii or related organisms showed increased ability to ferment RS3 compared to unsupplemented samples (Ze et al., 2012). Feeding a diet containing RS in humans significantly increases the presence of $R$. bromii and closely related organisms, further supporting their importance in RS metabolism (Abell et al., 2008). Martínez et al. (2010) confirmed the increases in $R$. bromii and E. rectale seen with RS2 and found that the effects of RS2 differed from those seen with RS4 in human subjects. Type 4 residual starch promoted the growth of Actinobacteria and Bacteroidetes but led to a decreased presence of Firmicutes while RS2 did not show phylum-level changes. At the species level, RS4 promoted the growth of $B$. adolescentis and Parabacteroides distasonis (Martínez et al., 2010). Resistant starch is particularly important, as its fermentation results in higher levels of butyric acid compared to the fermentation of other fibers (Huber et al., 2017).

Butyrate is important as it is used by colonocytes as an energy source, promotes barrier function, and reduces inflammation (Chen et al., 2017). Prevotella-rich microbiota produces higher levels of SCFAs through the metabolism of cereal grain fibers than microbiota rich in Bacteroides, and different organisms produce various levels and ratios of SCFAs from the same carbohydrate substrate in vitro assay (Chen et al., 2017). Therefore, the existing microbiota will influence how a substrate is metabolized. Both the fiber source and the existing microbiota impact how the microbiota will change in response to fiber consumption. Administration of three different fibers to microbiota dominated by Prevotella promoted the growth of the same Prevotella operational taxonomic unit (OTU) and resulted in similar SCFA profiles; however, administration of these three fibers to microbiota dominated by Bacteroides promoted different OTUs and resulted in different SCFA profiles (Chen et al., 2017).

\section{Protein}

A portion of dietary amino acids are catabolized in the small intestine where they are the primary energy source for enterocytes (Wu, 1998; Zoetendal et al., 2012). Evidence suggests that amino acid availability may be the limiting factor for microbial growth in the small intestine, stimulating microbial de novo synthesis (Zoetendal et al., 2012) and allowing amino acid exchange between the microbiota and their host to run in both directions (Davila et al., 2013; Zoetendal et al., 2012). Though amino acids can be taken up by the small intestine, it is generally accepted that they cannot be absorbed by the colon except for a short period after birth (Darragh et al., 1994). Many microorganisms are able to metabolize proteins, peptides and amino acids, with several being either preferential or obligate fermenters. Key proteolytic organisms in the colon include Bacteroides, Clostridium, Propionibacterium, Fusobacterium, Streptococcus, and Lactobacillus (Davila et al., 2013). Microorganisms differ in their abilities to metabolize proteins. Bacteroides vulgatus, Bacteroides distasonis, and Bacteroides ovatus secrete more proteases than other members of Bacteroides. These proteases have 
similar properties to those secreted by the host (Riepe et al., 1980). Microbial composition and proteolytic activity in the intestinal tract are important factors in gut health. Bacterial proteases are a possible link between intestinal dysbiosis and inflammatory conditions like IBD (Carroll and Maharshak, 2013). Proteases produced by Bacteroides reduced the activity of maltase and sucrase and were suggested to be capable of damaging brush border function in cases of bacterial overgrowth (Riepe et al., 1980). A metalloprotease from Enterococcus faecalis can also compromise gut barrier function and contribute to gut inflammation and the development of colitis in mice (Steck et al., 2011).

Some products of protein metabolism, such as nitrosamine precursors and trimethylamine $\mathrm{N}$-oxide (TMAO), can have detrimental effects on host health whereas others may confer health benefits (Kashantova et al., 2016). Colonic fermentation of aromatic amino acids can produce phenylpropanoids which can be beneficial to human health. Bacteroides thetaiotamicron, Bacteroides eggerthii, B. ovatus, Bacteroides fragilis, Parabacteroides distasonis, Eubacterium hallii, and Clostridium bartlettii can produce significant levels of phenylacetic acid, 4-hydroxylphenylacetic acid, and indole-3-acetic acid, and are likely the major source of these compounds in the colon (Russell et al., 2013). Though protein is an essential part of the diet, protein metabolites can be toxic and some evidence even links red meat to cardiovascular disease and colorectal cancer (Zhu et al., 2015).

A high-protein, low-carbohydrate diet in obese men was associated with decreased SCFAs, increased branched chain fatty acids, a large drop in Bacteroides, and a reduction in the major butyrate-producing Roseburial Eubacterium rectale group (Russell et al., 2011). Though there was no overall change in Lachnospiraceae or the second major group of butyrate-producing organisms related to $F$. prausnitzii, butyrate production was reduced under a high-protein diet (Russell et al., 2011). The drop in Bacteroides is counterintuitive, given that they have previously been listed as effective protein metabolizers. Mice fed a protein-deficient diet possessed microbiomes more similar to just-weaned mice than to mice of the same age fed a normal amount of protein and had increased excretion of metabolites, including the microbial metabolite choline and microbe-host cometabolites 3-indoxysulfate, $m$-hydroxyphenylpropionylsulfate, hippurate, and TMAO. Further, certain OTUs were associated with the excretion of specific metabolites, particularly Akkermansia muciniphila which was correlated with $N$-phenylacetylglycine, 3-indoxysulfate and trimethylamine, among other things (Mayneris-Perxachs et al., 2016). In contrast to the previous studies that found microbial changes associated with various levels of protein intake, Beaumont et al. (2017) found that a high-protein diet did not affect the microbiome of humans but did alter host gene expression and cause a shift in microbial metabolism toward amino acid degradation that varied with protein source.

Protein sources can be divided into meat, dairy, and plant-based sources. Dairy is often grouped either with meat to make plant-based and animal-based groupings, or with plant-based sources to make meat and non-meat groupings. In rats, meat-based sources lead to increased Firmicutes and fewer Bacteroidetes than non-meat proteins, with white meat having the highest level of Lactobacillus and an increased Firmicutes:Bacteroidetes ratio compared to other proteins (Zhu et al., 2015). Chicken is associated with elevated levels of Actinobacteria in rats and increased Bifidobacterium, and Bacteroides in vitro (Shen et al., 2010; Zhu et al., 2015). Red meat diets were associated with elevated levels of Ruminococcaceae and Lactobacillaceae, and beef increased Proteobacteria and Oscillibacter and decreased C. perfringens and C. histolyticum relative to chicken or fish after $30 \mathrm{~h}$ of fermentation (Shen et al., 2010; Zhu et al., 2015). Butteiger et al. (2016) compared the effects of milk protein isolate, soy protein isolate, and soy protein concentrate in hamsters and found that soy protein leads to higher microbial diversity and a shift in microbial metabolism. It was concluded that alterations in the microbiome may be the cause of the lipidlowering properties associated with soy (Butteiger et al., 2016). Soy, casein, and fish meal fed to rats did not induce microbial changes at the phylum level (An et al., 2014) At the family level, soy induced the highest levels of $R u$ minococcaceae and the lowest levels of Lactobacillaceae, and Bacteroidaceae was only present with fish meal (An et al., 2014). Lactobacillus was present with all three diets, however species varied based on which protein was fed; Lactobacillus intestinalis was present with casein, Lactobacillus reuteri was present with soy, and Lactobacillus murinis was present in those fed fish meal (An et al., 2014). Soy has also been found to increase Bacteroidetes in rats, and soy and casein both lead to increased levels of Lachnospiraceae (Zhu et al., 2015). While Butteiger et al. (2016) found that soy increased microbial diversity, Zhu et al. (2015) found that meat consumption increased microbial diversity compared to soy, concluding that animal-based proteins may create a better microbial composition than soy. Further, they conclude that meat proteins create a more balanced gut composition than non-meat proteins (Zhu et al., 2015). Discrepancies between the two studies could be due to species differences of hamsters versus rats or may be attributable to the formulation of the non-protein aspect of the diets. Regardless, the opposing conclusions warrant further investigation to determine how these dietary proteins may influence human health. 


\section{Fat}

A high-fat diet can alter the murine microbiome independent of obesity (Hildebrandt et al., 2009), and an altered microbiome characterized by a drop in Bacteroidetes and an increase in Firmicutes was linked with obesity in mice (Ley et al., 2005). Hildebrandt et al. (2009) found that Bacteroidetes abundance decreased upon administration of a high-fat diet irrespective of genetic predisposition to obesity and also noted a positive correlation between Actinobacteria and fat consumption (Hildebrandt et al., 2009). Krill oil increased microbial diversity compared to the reduced diversity created by a high-sugar, high-fat diet and helped to normalize the microbiota of mice fed a Western-type diet ( $\mathrm{Lu}$ et al., 2017). From a microbial perspective, long-term dairy and saturated fat intake led to significant increases in B. wadsworthia and a high-fat, animal-based diet showed increases in microbial DNA and RNA encoding sulfite reductases indicative of an elevated presence of B. wadsworthia in humans (David et al., 2013). A diet high in saturated fats creates an environment that promotes the expansion of this sulphite-reducing pathobiont and favors the onset of colitis in susceptible mice (Devkota et al., 2012). Omega-3 fish oil, on the other hand, appears to inhibit the growth of $B$. wadsworthia by inducing changes in the bile acid composition of mice (Devkota and Chang, 2015).

\section{Bile acid metabolism}

There is heavy interplay between diet, microbes, and bile metabolism. The enzymes produced by the intestinal microbiota are as important in bile acid metabolism as those encoded by the host's own genome (Long et al., 2017). In general, microbial interactions with bile acids increase the diversity and overall hydrophobicity of the bile acid pool, facilitating fecal elimination (Degirolamo et al., 2014). Microbes regulate both the synthesis and metabolism of bile acids, which in turn mold the intestinal microbial community by inhibiting the growth of bilesensitive bacteria and promoting the growth of bile metabolizing organisms like B. wadsworthia (David et al., 2013; Devkota et al., 2012). Bile acids regulate the gut microbiota both through the immune system by inducing the transcription of antimicrobial agents such as iNOS and IL-18 as well as through the direct antimicrobial effects of their detergent-like properties (Inagaki et al., 2006). Highfat diets in mice led to increased levels of enteric deoxycholic acid (DCA), a secondary bile acid that may be linked to obesity and liver cancer, through modulation of the gut microbiota (Yoshimoto et al., 2013). Increased DCA may inhibit certain members of Bacteroidetes and Firmicutes. Animal-based diets in humans result in increased fecal DCA and increased expression of genes encoding bile salt hydrolases, which break down bile acids into DCA (David et al., 2013). Organisms like Actinobacteria, Proteobacteria, Firmicutes, and Bacteroidetes possess hydroxysteroid dehydrogenases (HSDHs) and are able to generate oxo- or keto-bile acids in a reversible reaction that can result in epimerization (Wahlström et al., 2016). These iso-bile acids are found abundantly in the colon and feces, as well as in smaller levels in serum and urine, but are not found in the bile pool (Shefer et al., 1982; Wahlström et al., 2016). They may also be regulators of both the host metabolism and the gut microbial composition, as iso-DCA has been shown to favor the growth of Bacteroides in rats (Wahlström et al., 2016). Bacteria associated with the production of iso-bile acids include Eubacterium lentum, C. perfringens, and Ruminococcus gnavus (Wahlström et al., 2016), the last of which may be linked to IBD and lupus (Azzouz et al., 2019; Hall et al., 2017). Bile acid composition is therefore important not only in microbial community structure but also plays a significant role in host health, and there is a tight relationship between the type and level of fat consumed, bile acid makeup, and the gut microbiota.

\section{Unsaturated fatty acids}

Omega- 3 and omega- 6 fatty acids are polyunsaturated fatty acids that are an essential part of the diet. Omega- 6 fatty acids upregulate inflammation as they are precursors to pro-inflammatory signaling molecules, while omega- 3 fatty acids compete with this pathway and reduce inflammation (Robertson et al., 2018). Offspring of mice fed diets high in omega-3 displayed slightly higher levels of the anti-inflammatory cytokine IL-10, with reductions in peanut allergies but worsened reactions in certain bacterial infections (Myles et al., 2014). The traditional ratio of omega6:omega-3 consumed was likely close to $1: 1$, however the current ratio consumed in the Western diet is 10-50:1. The imbalance in this ratio may be a source of health problems, such as the metabolic endotoxemia and slight systemic inflammation associated with omega- 6 that are mitigated by omega-3 (Kaliannan et al., 2015; Robertson et al., 2018). The effect of omega- 3 on the gut microbiome has been extensively studied. Omega-3 induced changes in the gut microbial composition of mice which lead to decreased LPS production and intestinal permeability, both of which are increased in certain disease states (Kaliannan et al., 2015; Robertson et al., 2017).

In humans, omega-3 fatty acids and docosahexaenoic acid (DHA) levels correlated with alpha diversity of microbiome and increased Lachnospiraceae (Menni et al., 2017), and supplementation with omega-3 increased the butyrate-producing genera Bifidobacterium, Lachnospira, 
Roseburia, and Lactobacillus (Watson et al., 2018). In a murine model, maternal omega-3 supplementation reduced Lachnospiraceae, Anaerotruncus, and Roseburia and increased Blautia, Oscillibacter, Clostridiales, Robinsoniella, Lactococcus, and Eubacterium in offspring (Myles et al., 2014). Omega-3 fatty acid deficient mice displayed an elevated Firmicutes:Bacteroidetes ratio, while omega-3 supplemented mice had increased levels of Lactobacillus and Bifidobacterium (Robertson et al., 2017). Both human and animal studies have determined that omega-3 fatty acids affect the gut-brain axis through alterations in the gut microbiome. Omega- 3 fatty acid deficiency in early life has been related to impaired brain function, including psychomotor activity, cognition, attention, and vision, as well as psychological disorders such as depression, schizophrenia, and dementia (Robertson et al., 2017).

As with other aspects of diet, maternal consumption of omega-3 fatty acids influences the microbiome and disease states of her offspring later in life. These influences are apparent in several murine studies. Maternal fatty acid consumption during lactation had larger impacts on offspring than consumption during pregnancy (Robertson et al., 2018). Changes in dietary omega-3 fatty acids in the mother changed the levels of omega-3s in the mother's milk, and therefore in the offspring's diet (Robertson et al., 2017). Additionally, maternal fatty acid compositions were found to be transferrable to male, but not female, offspring (Robertson et al., 2018). Increased omega-3 fatty acid production correlated with reduced weight-gain and improved metabolism in offspring while reduced maternal omega-3s were linked to significant reductions in epsilonproteobacteria, Bacteroides, and Akkermansia, as well as an increase in Clostridia, indicating that microbial restructuring could be contributing to the phenotypic changes (Robertson et al., 2018). Myles et al. (2014) found that, compared to mice fed a low-fat diet, the offspring of mice fed high levels of omega-3 fatty acids displayed the reduced Bacteroidetes and increased ratio of Firmicutes:Bacteroidetes frequently associated with obesity, however they were not obese and did not have the increased inflammation typical of Western diets high in omega-6 fatty acids (Myles et al., 2014).

Conjugated linoleic acids (CLA), which are present mainly in meat and dairy, are a group of omega- 6 fatty acids that can reverse the problems associated with a highfat diet and promote the growth of Bacteroidetes, Prevotella, and A. muciniphila in mice (Chaplin et al., 2015). Trans-10, cis-12 CLA, which is found in dairy products, meat from ruminants, and is produced by organisms such as Lactobacillus plantarum PL62, demonstrates antiobesity effects but appears to induce hepatic steatosis and hyperinsulinemia, both of which are observed in diabetic or obese individuals (Lee et al., 2007; Marques et al., 2015). In mice, trans-10, cis-12 CLA reduced the Firmicutes:Bacteroidetes ratio and decreased levels of Desulfovibrionaceae, Lachnospiraceae, Peptococcaceae, and Clostridiales Family XIII. Incertae Sedis while increasing Porphyromonadaceae (Marques et al., 2015).

The impact of the unsaturated:saturated fatty acid ratio on the gut microbiome and human health has been investigated through the use of various plant and animal oils. De Wit et al. (2012) focused on the different effects associated with low, roughly equal, and high ratios of polyunsaturated to saturated fatty acids in high-fat diets. High-fat palm oil, olive oil, and safflower oil diets were compared to a low-fat palm oil diet in mice. High-fat palm oil and high-fat olive oil diets led to increase obesity despite a lack of change in the gut microbiota between the high fat olive oil diet and the control. The levels of Deferribacteres varied with each diet, but the microbiota associated with the control, olive oil, and safflower oil diets did not otherwise differ. A highfat palm oil diet reduced microbial diversity and increased the Firmicutes:Bacteroidetes ratio, especially with respect to Clostridium clusters XI, XVII, and XVIII (de Wit et al., 2012). Hidalgo et al. (2014) found an increased level of Bacteroidetes in mice fed olive oil compared to those fed palm oil (Hidalgo et al., 2014). The fatty acid profile of olive oil can vary widely, as can the phenolic content. Extra virgin and refined olive oil exhibited the microbiota more similarly to each other than to butter, which showed an elevated Firmicutes:Bacteroidetes ratio and a significant increase in Lactobacillaceae. The microbial profile associated with refined olive oil was between that of butter and extra virgin olive oil, which is interesting given that the only difference between the olive oils used in the study was the phenolic content (Hidalgo et al., 2014).

\section{Micronutrients and heavy metals}

\section{Vitamins and minerals}

Micronutrients are compounds that are vital in small amounts for proper development and function but in large doses often become toxic. Deficiency in the vitamin A metabolite retinoic acid impairs immunity while excess can lead to inflammatory disorders (Lee and Ko, 2016). Micronutrients can be classified as vitamins, which are organic, and minerals, which are inorganic. While macronutrients are broken down and used in energy production and anabolism, micronutrients largely function as enzymatic cofactors in biochemical reactions. Prokaryotes require micronutrients just as eukaryotes do, however unlike animals and humans which need to obtain both vitamins and minerals through their diet, prokaryotes are 
able to synthesize certain vitamins. There is debate regarding the bioavailability of vitamins produced by the gut microbiota, and the answer likely depends on which region of the gut various compounds are synthesized in. Nearly all vitamin absorption takes place in the small intestine, however some may occur in the colon as well (Biesalski, 2016). Thus, the gut microbiota both influence and are influenced by levels of micronutrients.

\section{Vitamins}

Vitamins have a significant impact on the immune system and intestinal inflammatory diseases are often associated with vitamin deficiency (Biesalski, 2016; Jin et al., 2015). Vitamin A, for example, was found to be anti-viral against norovirus infection (Lee and Ko, 2016) and vitamin D is known to benefit immune and cardiovascular function. Vitamin A had a larger impact on gut microbial community structure and gene expression than folate, iron, or zinc (Hibberd et al., 2017). Vitamin D deficiency and vitamin D receptor (VDR) downregulation are associated with inflammatory and autoimmune diseases (Bashir et al., 2016; Jin et al., 2015). Norovirus-infected mice supplemented with retinoic acid exhibited increased Lactobacillus, whereas norovirus infection alone resulted in decreased Lactobacillaceae populations. Lactobacillus and retinoic acid each were found to have independent anti-viral activity against norovirus (Lee and Ko, 2016). Supplementation with retinoic acid in the absence of norovirus infection increased Allobaculum, Aggregatibacter, Bifdobacterium, Dialister, and Enhydrobacter in mice (Lee and Ko, 2016).

In humans, vitamin D supplementation decreased the relative abundance of gammaproteobacteria such as Pseudomonas, Escherichia, and Shigella and increased bacterial richness in the upper gastrointestinal tract but did not result in microbial changes downstream of the terminal ileum (Bashir et al., 2016). Vitamin D receptors also regulate antimicrobial peptide expression which can impact the microbiome, and microbial byproducts such as secondary bile acids may be able to activate VDRs (Clark and Mach, 2016; Jin et al., 2015). VDR knockout mice had lower levels of Lactobacillus and increased Clostridium and Bacteroides in their feces and decreased Alistipes and Odoribacter with increased Eggerthella in their ceca (Jin et al., 2015). Another mouse study found that vitamin D increases beneficial microorganisms, including Lactobacillaceae, and protects against induced colitis (Ooi et al., 2013). It is also hypothesized that supplying different ratios of corrinoids, the family of compounds to which vitamin $\mathrm{B}_{12}$ belongs, can shape the microbiome as different microbes require specific corrinoids (Degnan et al., 2014), though this remains to be tested.
Intestinal microbes can also produce many vitamins including vitamin $\mathrm{K}_{2}$ (menaquinone), $\mathrm{B}_{12}$ (cobalamin), and biotin. Different organisms are capable of producing specific vitamins-for example, certain members of Bifidobacteria can produce folate (LeBlanc et al., 2013). The bioavailability of microbially produced vitamin $\mathrm{K}$ is questionable as absorption occurs in the small intestine while production presumably occurs in the large intestine (Biesalski, 2016). To be host-available, production of a compound should occur at or upstream of the absorption site. Zoetendal et al. (2012) investigated the small intestinal microbiota by use of ileostomists, individuals who have had their colon removed, and discovered increased synthesis of cobalamin and biotin in ileostomy samples compared to fecal samples. (Zoetendal et al., 2012). Genes related to cobalamin and biotin synthesis were associated mostly with Proteobacteria but were also linked to Firmicutes and Bacteroidetes (Zoetendal et al., 2012). Since most biotin absorption occurs in the small intestine, it was determined that microbes may supply a significant level of biotin to their hosts (Zoetendal et al., 2012). Some watersoluble vitamins, including biotin, can also be taken up by colonocytes (Biesalski, 2016). Conversely, microbially derived cobalamin is not likely shared with human hosts and microbes more likely compete with the host for dietary cobalamin (Biesalski, 2016; Degnan et al., 2014).

Most of the microorganisms present in the intestinal tract lack the genes for cobalamin production and therefore require exogenous cobalamin (Degnan et al., 2014). Further, cobalamin produced in the colon where microbial load is highest is not bioavailable as uptake occurs in the small intestine (Degnan et al., 2014). While fewer organisms are present in the small intestine, Zoetendal et al. (2012) found genes related to cobalamin and biotin synthesis to be overrepresented in ileostomy samples (Zoetendal et al., 2012). There is a disconnect between microbiome data and information on vitamin absorption, as the vast majority of vitamin absorption occurs in the small intestine but most microbiome studies are performed on fecal samples. Further studies investigating vitamin production and absorption along the intestinal tract, specifically in the small intestine, are required to understand the bioavailability of microbially-produced vitamins such as menaquinone and cobalamin. Even if the microbiallysynthesized vitamins produced inside the intestinal tract are not host-available, microbes can still impact vitamin intake through their application in the food industry. Since different organisms use and produce different levels of vitamins, vitamin levels in fermented foods can be manipulated through selection of starter cultures (LeBlanc et al., 2013). While vitamin levels can be manipulated through the use of specific taxa, mineral content cannot be influenced this way. 


\section{Minerals}

Fewer studies are available on the effects of minerals on the gut microbiome. Mineral deficiencies are prevalent throughout the world, particularly in low-resource areas, and can result in pathologies ranging from iron deficiency anemia to various enteropathies (Mayneris-Perxachs et al., 2016; Tang et al., 2017). Mineral excess can also result in gastrointestinal distress, making it important to understand the impact that minerals have on the gut microbiota. Mayneris-Perxachs et al. (2016) found that the microbiomes of zinc-deficient mice were comparable to their well-nourished counterparts (Mayneris-Perxachs et al., 2016). Zinc oxide was found to modulate the microbiome of piglets in a manner similar to antibiotics, possibly explaining why zinc supplementation is effective in preventing and treating diarrhea in piglets (Yu et al., 2017). Antibiotics and zinc both resulted in changes to the nonpredominant microbiota of the ileal digesta and colon ( $\mathrm{Yu}$ et al., 2017). In the ileal digesta, zinc and antibiotic supplementation at a pharmacological level increased Spirochaetes, Tenericutes, Euryarchaeota, Verrucomicrobia, TM7 and Enterobacteriales, and decreased Chlamydiae and Campylobacterales (Yu et al., 2017). Zinc also decreased the amount of antibiotics necessary to induce Clostridium difficile infection, a common cause of antibiotic-associated diarrhea and other nosocomial infections (Zackular et al., 2016). Microbial changes included decreased diversity, decreased Turicibacter and Clostridium, and increased Enterococcus and Clostridium XI (Zackular et al., 2016). Micronutrient deficiencies such as zinc deficiency can increase susceptibility to the effects of toxin exposure, for example lead or arsenic (Bushnell and Levin, 1983; Gaulke et al., 2018).

Iron deficiency is one of the most prevalent micronutrient deficiencies, however iron supplementation is often associated with adverse effects (Tang et al., 2017). Excessive iron intake can promote the growth of pathogenic strains of organisms such as Salmonella, Shigella, and E. coli, and multiple studies cited by Tang et al. (2017) have found that iron supplementation or fortification can result in unfavorable gut microbiomes, for example with depleted Bifidobacterium and increased pathogens, and increased gut inflammation in children and infants (Tang et al., 2017). Tang et al. (2017) found that, while iron supplementation did not cause an increase in E. coli and Shigella, it did prevent their normal decrease in infants (Tang et al., 2017). Since nutrition can significantly impact the intestinal microbiota and dysbiosis has been correlated with a range of afflictions including psychiatric disorders, Winther et al. (2015) tested the hypothesis that imbalances in the gut-brain axis induced by magnesium deficiency could lead to depressive-like behavior. Mice fed a magnesium deficient diet had changes in their gut microbiome that positively correlated with hippocampal IL-6, and expressed increased depressive-like behavior (Winther et al., 2015). Specific taxa were not investigated, leaving the potential role that micronutrients play in the gut-brain axis largely unexplored. While micronutrients are organic and inorganic compounds essential to proper cell function, organic and inorganic toxicants and contaminants which make their way into the food supply can impede function and be a detriment to human health.

\section{Heavy metals}

Heavy metals are inorganic compounds that exist in the environment and often make their way into the food supply, with levels ranging from low concentrations to above safe limits, as is the case in the vegetables, meat, and fish in a mining city in China (Cheng et al., 2017). This city is not an isolated occurrence; heavy metals such as cadmium, arsenic, lead, and mercury have all been found in marine fish (Bosch et al., 2016). The level of concern varies based on the rates of bioaccumulation in different tissues and the form of the compound present.

\section{Methylmercury}

Methylmercury $(\mathrm{MeHg})$ is the most widely known contaminant of fish and can be a major concern with the consumption of certain species, especially for vulnerable populations including children and women who are pregnant or nursing. Gut microbes can modulate mercury toxicity through methylation and demethylation and may be able to decrease $\mathrm{MeHg}$ bioavailability (Lin et al., 2020; Rothenberg et al., 2016). Little difference was seen between the microbiota of human subjects with high stool $\mathrm{MeHg}$ compared to low, and any change in diversity was attributed to rare taxa (Rothenberg et al., 2016). Methylmercury treated rats presented with decreased Bacteroidetes and Proteobacteria and increased Firmicutes (Lin et al., 2020). Conversely, methylmercury increased Bacteroidetes and decreased Firmicutes in mice and resulted in genus and species-level changes including increased Prevotella, Odoribacter, and Lactobacillus ruminis (Zhang et al., 2019). In rats, MeHg exposure decreased Lactobacillaceae, Bacteroidaceae, Streptococcaceae, and Sutterellaceae, increased Desulfovibrionaceae, Helicobacteraceae, Peptococcaceae, and Rhodospirillaceae, and negatively impacted the gut-brain axis (Lin et al., 2020). 


\section{Cadmium}

Not all heavy-metal contaminants are as well-known as Methylmercury. Cadmium is a lesser-known threat that impacts the global population (Ba et al., 2017). Many regions have an average weekly cadmium intake at or above tolerable levels. Early life exposure in male mice reduced microbial diversity, altered microbial composition, and contributed to adult adiposity even in cases where the microbiota fully recovered. Microbial effects included increased Bacteroidetes and decreased Firmicutes as well as reduced Bifidobacterium and Prevotella and increased Sphingomonas (Ba et al., 2017). Zhang et al. (2015) also found that cadmium decreases Firmicutes and Gammaproteobacteria, and increases Bacteroidetes (Zhang et al., 2015).

\section{Lead and arsenic}

Similar to cadmium, lead has been linked to body weight gain and obesity (Jin et al., 2017). Microbial changes associated with lead intake include an increased Firmicutes:Bacteroidetes ratio, increased Desulfovibrionaceae, Barnesiella, and Clostridium XIVb, and decreased Caulobacterales and A. muciniphila (Jin et al., 2017). Arsenic in fish is less of a concern as up to $90 \%$ of the arsenic measured in fish is in the less toxic organic form (Bosch et al., 2016), however arsenic is regularly encountered by a large portion of the global population through food and drinking water (Gaulke), the latter of which often contains the more toxic inorganic form (Bosch et al., 2016; Jin et al., 2017). Arsenic has been linked to a decrease in Firmicutes and an increase in Bacteroidetes (Jin et al., 2017). Arsenic exposure and zinc deficiency, which are comorbid in a large portion of the population, have independent impacts on the gut microbiota and together act synergistically (Gaulke et al., 2018). These microbial changes were linked to physiological changes, which is expected as the gut microbiome modulates host interactions with xenobiotics and changing the structure could change host toxin exposure (Gaulke et al., 2018).

Acknowledgements This review paper was partially supported by the BUILD (Building University-Industry linkages through Learning and Discovery) Dairy Grant and the KFRI (Korea Food Research Institute) awarded to Si Hong Park.

\section{Compliance with ethical standards}

Conflict of interest The authors declared that they do not have conflict of interest.

Open Access This article is licensed under a Creative Commons Attribution 4.0 International License, which permits use, sharing, adaptation, distribution and reproduction in any medium or format, as long as you give appropriate credit to the original author(s) and the source, provide a link to the Creative Commons licence, and indicate if changes were made. The images or other third party material in this article are included in the article's Creative Commons licence, unless indicated otherwise in a credit line to the material. If material is not included in the article's Creative Commons licence and your intended use is not permitted by statutory regulation or exceeds the permitted use, you will need to obtain permission directly from the copyright holder. To view a copy of this licence, visit http://creativecommons. org/licenses/by/4.0/.

\section{References}

Abell GCJ, Cooke CM, Bennett CN, Conlon MA, McOrist AL. Phylotypes related to Ruminococcus bromii are abundant in the large bowel of humans and increase in response to a diet high in resistant starch. FEMS Microbiol. Ecol. 66: 505-515 (2008)

An C, Kuda T, Yazaki T, Takahashi H, Kimura B. Caecal fermentation, putrefaction and microbiotas in rats fed milk casein, soy protein or fish meal. Appl. Microbiol. Biotechnol. 98: 2779-2787 (2014)

Azzouz D, Omarbekova A, Heguy A, Schwudke D, Gisch N, Rovin BH, Caricchio R, Buyon JP, Alekseyenko AV, Silverman GJ. Lupus nephritis is linked to disease-activity associated expansions and immunity to a gut commensal. Ann. Rheum. Dis. 78: 946-947 (2019)

Ba Q, Li M, Chen P, Huang C, Duan X, Lu L, Li J, Chu R, Xie D, Song H, Wu Y, Ying H, Jia X, Wang H. Sex-dependent effects of cadmium exposure in early life on gut microbiota and fat accumulation in mice. Environ. Health Perspect. 125: 437-446 (2017)

Bashir M, Prietl B, Tauschmann M, Mautner SI, Kump PK, Treiber G, Wurm P, Gorkiewics G, Högenauer C, Pieber TR. Effects of high doses of vitamin $\mathrm{D}_{3}$ on mucosa-associated gut microbiome vary between regions of the human gastrointestinal tract. Eur. J. Nutr. 55: 1479-1489 (2016)

Beaumont M, Portune KJ, Steuer N, Lan A, Cerrudo V, Audebert M, Dumont F, Mancano G, Khodorova N, Andriamihaja M, Airinei G, Tomé M, Benamouzig R, Davila AM, Claus SP, Sanz Y, Blachier F. Quantity and source of dietary protein influence metabolite production by gut microbiota and rectal mucosa gene expression: a randomized, parallel, double-blind trial in overweight humans. Am. J. Clin. Nutr. 106: 1005-1019 (2017)

Biesalski HK. Nutrition meets the microbiome: micronutrients and the microbiota. Ann. N. Y. Acad. Sci. 1372: 53-64 (2016)

Bosch AC, O'Neill B, O Sigge G, Kerwath SE, Hoffman LC. Heavy metals in marine fish meat and consumer health: a review. J. Sci. Food Agric. 96: 32-48 (2016)

Bushnell PJ, Levin ED. Effects of zinc deficiency on lead toxicity in rats. Neurotoxicol. Teratol. 5: 283-288 (1983)

Butteiger DN, Hibberd AA, McGraw NJ, Napawan N, Hall-Porter M, Krul ES. Soy protein compared with milk protein in a western diet increases gut microbial diversity and reduces serum lipids in golden Syrian hamsters. J. Nutr. 146: 697-705 (2016)

Carroll IA, Maharshak N. Enteric bacterial proteases I inflammatory bowel disease- pathophysiology and clinical implications. World J. Gastroenterol. 19: 7531-7543 (2013)

Chaplin A, Parra P, Serra F, Palou A. Conjugated linoleic acid supplementation under a high-fat diet modulates stomach protein expression and intestinal microbiota in adult mice. PLoS One 10: e0125091 (2015)

Chen T, Long W, Zhang C, Liu S, Zhao L, Hamaker BR. Fiberutilizing capacity varies in Prevotella- versus Bacteroidesdominated gut microbiota. Sci. Rep. 7: 1 (2017) 
Cheng J, Zhang X, Tang Z, Yang Y, Nie Z, Huang Q. Concentrations and human health implications of heavy metals in market foods from a Chinese coal-mining city. Environ. Toxicol. Pharmacol. 50: 37-44 (2017)

Clark A, Mach N. Role of vitamin D in the hygiene hypothesis: the interplay between vitamin $\mathrm{D}$, vitamin $\mathrm{D}$ receptors, gut microbiota, and immune response. Front. Immunol. 7: 627 (2016)

Clarke G, Stilling RM, Kennedy PJ, Stanton C, Cryan JF, Dinan TG. Minireview: gut microbiota: the neglected endocrine organ. Mol. Endocrinol. 28: 1221-1238 (2014)

Dahlin M, Prast-Nielsen S. The gut microbiome and epilepsy. EBioMedicine. 44: 741-746 (2019)

Darragh AJ, Cranwell PD, Moughan PJ. Absorption of lysine and methionine from the proximal colon of the piglet. Br. J. Nutr. 71: 739-752 (1994)

David LA, Maurice CF, Carmody RN, Gootenberg DB, Button JE, Wolfe BE, Ling AV, Devlin AS, Varma Y, Fischbach MA, Biddinger SB, Dutton RJ, Turnbaugh PJ. Diet rapidly and reproducibly alters the human gut microbiome. Nature 505: 559-563 (2013)

Davila AM, Blachier F, Gotteland M, Andriamihaja M, Benetti PH, Sanz Y, Tomé D. Re-print of "Intestinal luminal nitrogen metabolism: role of the gut microbiota and consequences for the host." Pharmacol. Res. 69: 114-126 (2013)

Degirolamo C, Rainaldi S, Bovenga F, Murzilli S, Moschetta A. Microbiota modification with probiotics induces hepatic bile acid synthesis via downregulation of the Fxr-Fgf15 axis in mice. Cell Rep. 7: 12-18 (2014)

Degnan PH, Taga ME, Goodman AL. Vitamin $\mathrm{B}_{12}$ as a modulator of gut microbial ecology. Cell Metab. 20: 769-778 (2014)

Devkota S, Wang Y, Musch MW, Leone V, Fehlner-Peach H, Nadimpalli A, Antonopoulos DA, Jabri B, Chang EB. Dietaryfat-induced taurocholic acid promotes pathobiont expansion and colitis in Il10 ${ }^{-/}$mice. Nature 487: 104-109 (2012)

Devkota S, Chang EB. Interactions between diet, bile acid metabolism, gut microbiota, and inflammatory bowel diseases. Dig. Dis. 33: 351-356 (2015)

de Wit N, Derrien M, Bosch-Vermeulen H, Oosterink E, Keshtkar S, Duval C, de Vogel-van den Bosch J, Kleerebezem M, Müller M, van der Meer R. Saturated fat stimulates obesity and hepatic steatosis and affects gut microbiota composition by an enhanced overflow of dietary fat to the distal intestine. Am. J. Physiol. Gastrointest. Liver Physiol. 303: G589-G599 (2012)

Didienne R, Defargues C, Callon C, Meylheuc T, Hulin S, Montel MC. Characteristics of microbial biofilm on wooden vats ('gerles') in PDO Salers cheese. Int. J. Food Microbiol. 156: 91-101 (2012)

Eckburg PB, Bik EM, Bernstein CN, Purdom E, Dethlefsen L, Sargent M, Gill SR, Nelson KE, Relman DA. Diversity of the human intestinal microbial flora. Science 308: 1635-1638 (2005)

Gaulke CA, Rolshoven J, Wong CP, Hudson LG, Ho E, Sharpton TJ. Marginal zinc deficiency and environmentally relevant concentrations of arsenic elicit combined effects on the gut microbiome. mSphere 3: e00521-18 (2018)

Gong L, Cao W, Chi H, Wang J, Zhang H, Liu J, Sun B. Whole cereal grains and potential health effects: involvement of the gut microbiota. Food Res. Int. 103: 84-102 (2018)

Graf D, Di Cagno R, Fåk F, Flint HJ, Nyman M, Saarela M, Watzl B. Contribution of diet to the composition of the human gut microbiota. Microb. Ecol. Health Dis. 26: 26164 (2015)

Hall AB, Yassour M, Sauk J, Garner A, Jiang X, Arthur T, Lagoudas GK, Vatanen T, Fornelos N, Wilson R, Bertha M, Cohen M, Garber J, Khalili H, Gevers D, Ananthakrishnan AN, Kugathasan $\mathrm{S}$, Lander ES, Blainey $\mathrm{P}$, Vlamakis $\mathrm{H}$, Xavier RJ, Huttenhower C. A novel Ruminococcus gnavus clade enriched in inflammatory bowel disease patients. Genome Med. 9: 103 (2017)

Heiman ML, Greenway FL. A healthy gastrointestinal microbiome is dependent on dietary diversity. Mol. Metab. 5: 317-320 (2016)

Hibberd MC, Wu M, Rodionov DA, Li X, Cheng J, Griffin NW, Barratt MJ, Giannone RJ, Hettich RL, Osterman AL, Gordon JI. The effects of micronutrient deficiencies on bacterial species from the human gut microbiota. Sci. Transl. Med. 9: eaal4069 (2017)

Hidalgo M, Prieto I, Abriouel H, Cobo A, Benomar N, Gálvez A, Martinez-Cañamero M. Effect of virgin and refined olive oil consumption on gut microbiota. Comparison to butter. Food Res. Int. 64: 553-559 (2014)

Hildebrandt MA, Hoffmann C, Sherrill-Mix SA, Keilbaugh SA, Hamady M, Chen YY, Knight R, Ahima RS, Bushman F, Wu GD. High-fat diet determines the composition of the murine gut microbiome independently of obesity. Gastroenterology 137: 1716-1724 (2009)

Huber KC, BeMiller JN. Carbohydrates. pp. 91-169. In: Fennema's Food Chemistry Fifth Edition. Damodaran S, Parkin KL (ed). CRC Press, Inc., Boca Raton, FL, USA (2017)

Inagaki T, Moschettat A, Lee YK, Peng L, Zhao G, Downes M, Yu RT, Shelton JM, Richardson JA, Repa JJ, Mangelsdorf DJ, Kliewer SA. Regulation of antibacterial defense in the small intestine by the nuclear bile acid receptor. Proc. Natl. Acad. Sci. U.S.A. 103: 3920-3925 (2006)

Jandhyala SM, Talukdar R, Subramanyam C, Vuyyuru H, Sasikala M, Reddy DN. Role of the normal gut microbiota. World J. Gastroenterol. 21: 8787-8803 (2015)

Jefferson A, Adolphus K. The effects of intact cereal grain fibers, including wheat bran on the gut microbiota composition of healthy adults: a systematic review. Front. Nutr. 6: 33 (2019)

Jin D, Wu S, Zhang Y, Lu R, Xia Y, Dong H, Sun J. Lack of vitamin $\mathrm{D}$ receptor causes dysbiosis and changes functions of the murine intestinal microbiome. Clin. Ther. 37: 996-1009 (2015)

Jin Y, Wu S, Zeng Z, Fu Z. Effects of environmental pollutants on gut microbiota. Environ. Pollut. 222: 1-9 (2017)

Kaliannan K, Wang B, Li XY, Kim KJ, Kang JX. A host-microbiome interaction mediates the opposing effects of omega- 6 and omega-3 fatty acids on metabolic endotoxemia. Sci. Rep. 5: $11276(2015)$

Kamada N, Chen GY, Inohara N, Núñez G. Control of pathogens and pathobionts by the gut microbiota. Nat. Immunol. 14: 685-690 (2013)

Kashantova DA, Popenko AS, Tkacheva ON, Tyakht AB, Alexeev DG, Boytsov SA. Association between the gut microbiota and diet: fetal life, early childhood, and further life. Nutrition 32: 620-627 (2016)

Kristek A, Wiese M, Heuer P, Kosik O, Schar MY, Soycan G, Alsharif S, Kuhnle GGC, Walton G, Spencer JPE. Oat bran, but not its isolated bioactive $\beta$-glucans or polyphenols, have a bifidogenic effect in an in vitro fermentation model of the gut microbiota. Br. J. Nutr. 121: 549-559 (2019)

LeBlanc JG, Milani C, Savoy de Giori G, Sesma F, van Sinderen D, Ventura M. Bacteria as vitamin suppliers to their host: a gut microbiota perspective. Curr. Opin. Biotech. 24: 160-168 (2013)

Lee $\mathrm{H}, \mathrm{Ko} \mathrm{G}$. Antiviral effect of vitamin A on norovirus infection via modulation of the gut microbiome. Sci. Rep. 6: 1 (2016)

Lee K, Paek K, Lee H, Park J, Lee Y. Antiobesity effect of trans-10, cis-12-conjugated linoleic acid-producing Lactobacillus plantarum PL62 on diet-induced obese mice. J. Appl. Microbiol. 103: 1140-1146 (2007)

Ley RE, Bäckhed F, Turnbaugh P, Lozupone CA, Knight RD, Gordon JI. Obesity alters gut microbial ecology. Proc. Natl. Acad. Sci. U.S.A. 102: 11070-11075 (2005) 
Lin X, Zhao J, Zhang W, He L, Wang L, Chang D, Cui L, Gao Y, Li $\mathrm{B}$, Chen C, Li YF. Acute oral methylmercury exposure perturbs the gut microbiome and alters gut-brain axis related metabolites in rats. Ecotoxicol. Environ. Saf. 190: 110130 (2020)

Long SL, Gahan CG, Joyce SA. Interactions between gut bacteria and bile in health and disease. Mol. Asp. Med. 56: 54-65 (2017)

Lu C, Sun T, Li Y, Zhang D, Zhou J, Su X. Modulation of the gut microbiota by krill oil in mice fed a high-sugar high-fat diet. Front. Microbiol. 8: 905 (2017)

Maier TV, Lucio M, Lee LH, VerBerkmoes NC, Brislawn CJ, Bernhardt J, Lamendella R, McDermott JE, Bergeron N, Heinzmann SS, Morton JT, González A, Ackermann G, Knight R, Riedel K, Krauss RM, Schmitt-Kopplin P, Jansson JK. Impact of dietary resistant starch on the human gut microbiome, metaproteome, and metabolome. mBio 8: e01343-17 (2017)

Marques RM, Wall R, O'Sullivan O, Fitzgerald GF, Shanahan F, Quigley EM, Cotter PD, Cryan JF, Dinan TG, Ross RP, Stanton C. Dietary trans-10, cis-12-conjugated linoleic acid alters fatty acid metabolism ad microbiota composition in mice. Br. J. Nutr. 113: $728-738$ (2015)

Martínez I, Kim J, Duffy PR, Schlegel VL, Walter J. Resistant starches types 2 and 4 have differential effects on the composition of the fecal microbiota in human subjects. PLoS One 5: e15046 (2010)

Mayneris-Perxachs J, Bolick DT, Leng J, Medlock GL, Kolling GL, Papin JA, Swann JR, Guerrant RL. Protein- and zinc-deficient diets modulate the murine microbiome and metabolic phenotype. Am. J. Clin. Nutr. 104: 1253-1262 (2016)

Mendis M, Leclerc E, Simsek S. Arabinoxylans, gut microbiota and immunity. Carbohydr. Polym. 139: 159-166 (2016)

Menni C, Zierer J, Pallister T, Jackson MA, Long T, Mohney RP, Steves CJ, Spector TD, Valdes AM. Omega-3 fatty acids correlate with gut microbiome diversity and production of $\mathrm{N}$-carbamylglutamate in middle aged and elderly women. Sci. Rep. 7: 1 (2017)

Metzler-Zebeli BU, Zijlstra RT, Mosenthin R, Gänzle MG. Dietary calcium phosphate content and oat $\beta$-glucan influence gastrointestinal microbiota, butyrate-producing bacteria and butyrate fermentation in weaned pigs. FEMS Microbiol. Ecol. 75: 402-413 (2011)

Myles IA, Pincus NB, Fontecilla NM, Datta SK. Effects of parental omega-3 fatty acid intake on offspring microbiome and immunity. PLoS One 9: e87181 (2014)

Niederwerder MC. Fecal microbiota transplantation as a tool to treat and reduce susceptibility to disease in animals. Vet. Immunol. Immunopathol. 206: 65-72 (2018)

O'Hara AM, Shanahan F. The gut flora as a forgotten organ. EMBO Rep. 7: 393-688 (2006)

Ooi JH, Li Y, Rogers CJ, Cantorna MT. Vitamin D regulates the gut microbiome and protects mice from dextran sodium sulfateinduced colitis. J. Nutr. 143: 1679-1686 (2013)

Riepe SP, Goldstein J, Alpers DH. Effect of secreted Bacteroides proteases on human intestinal brush border hydrolases. J. Clin. Invest. 66: 314-322 (1980)

Robertson RC, Oriach CS, Murphy K, Moloney GM, Cryan JF, Dinan TG, Ross RP, Catherine S. Omega-3 polyunsaturated fatty acids critically regulate behavior and gut microbiota development in adolescence and adulthood. Brain Behav. Immun. 59: 21-37 (2017)

Robertson RC, Kaliannan K, Strain CR, Ross RP, Stanton C, Kang JX. Maternal omega-3 fatty acids regulate offspring obesity through persistent modulation of gut microbiota. Microbiome 6: 95 (2018)

Rothenberg SE, Keiser S, Ajami NJ, Wong MC, Gesell J, Petrosino $\mathrm{JF}$, Johs A. The role of gut microbiota in fetal methylmercury exposure: insights from a pilot study. Toxicol. Lett. 242: 60-67 (2016)

Russell WR, Gratz SW, Duncan SH, Holtrop G, Ince J, Scobbie L, Duncan G, Johnstone AM, Lobley GE, Wallace RJ, Duthie GG, Flint HJ. High-protein, reduced-carbohydrate weight-loss diets promote metabolite profiles likely to be detrimental to colonic health. Am. J. Clin. Nutr. 93: 1062-1072 (2011)

Russell WR, Duncan SH, Scobbie L, Duncan G, Cantlay L, Calder AG, Anderson SE, Flint HJ. Major phenylpropanoid-derived metabolites in the human gut can arise from microbial fermentation of protein. Mol. Nutr. Food Res. 57: 523-535 (2013)

Sandoval DA, Seeley RJ. The microbes made me eat it. Science 328: 179-180 (2010)

Shefer S, Salen G, Hauser S, Dayal B, Batta AK. Metaboism of isobile acids in the rat. J. Biol. 257: 1401-1406 (1982)

Shen Q, Chen YA, Tuohy KM. A comparative in vitro investigation into the effects of cooked meats on the human faecal microbiota. Anaerobe 16: 572-577 (2010)

Shen RL, Dang XY, Dong JL, Hu XZ. Effects of oat $\beta$-glucan and barley $\beta$-glucan on fecal characteristics, intestinal microflora, and intestinal bacterial metabolites in rats. J. Agric. Food Chem. 60: 11301-11308 (2012)

Snoeyenbos GH, Weinack OM, Smyser CF. Mixture to protect poultry from Salmonella. United States of America 4,335,107 (1982)

Sommer F, Bäckhed F. The gut microbiota-masters of host development and physiology. Nat. Rev. Microbiol. 11: 227-238 (2013).

Sonnenburg ED, Sonnenburg JL. Starving our microbial self: the deleterious consequences of a diet deficient in microbiotaaccessible carbohydrates. Cell Metab. 20: 779-786 (2014)

Sonnenburg ED, Smits SA, Tikhonov M, Higginbottom SK, Wingreen NS, Sonnenburg JL. Diet-induced extinctions in the gut microbiota compound over generations. Nature 529: 212-215 (2016)

Steck N, Hoffmann M, Sava IG, Kim SC, Hahne H, Tonkonogy SL, Mair K, Krueger D, Pruteanu M, Shanahan F, Vogelmann R, Schemann M, Kuster B, Sartor RB, Haller D. Enterococcus faecalis metalloprotease compromises epithelial barrier and contributes to intestinal inflammation. Gastroenterology 141: 959-971 (2011)

Tang M, Frank DN, Hendricks AE, Ir D, Esamai F, Liechty E, Hambidge KM, Krebs NF. Iron in micronutrient powder promotes an unfavorable gut microbiota in Kenyan infants. Nutrients 9: 776 (2017)

Van den Abbeele P, Koen V, Van de Wiele T, Verstraete W, Possemiers S. Different human gut models reveal the distinct fermentation patterns of arabinoxylan versus inulin. J. Agric. Food Chem. 61: 9819-9827 (2013)

Vitaglione P, Mazzone G, Lembo V, D’Argenio G, Rossi A, Guido M, Savoia M, Salomone F, Mennella I, De Filippis F, Ercolini D, Caporaso N, Morisco F. Coffee prevents fatty liver disease induced by a high-fat diet by modulating pathways of the gutliver axis. J. Nutr. Sci. 8: e15 (2019)

Vuong HE, Hsiao EY. Emerging roles for the gut microbiome in autism spectrum disorder. Biol. Psychiatry 81: 411-423 (2017)

Wahlström A, Sayin SI, Marschall HU, Bäckhed F. Intestinal crosstalk between bile acids and microbiota and its impact on host metabolism. Cell Metab. 24: 41-50 (2016)

Wang Y, Ames NP, Tun HM, Tosh SM, Jones PJ, Khafipour E. High molecular weight barley $\beta$-glucan alters gut microbiota toward reduced cardiovascular disease risk. Front. Microbiol. 7: 129 (2016)

Watson H, Mitra S, Croden FC, Taylor M, Wood HM, Perry SL, Spencer JA, Quirke P, Toogood GJ, Lawton CL, Dye L, Loadman PM, Hull MA. A randomised trial of the effect of 
omega-3 polyunsaturated fatty acid supplements on the human intestinal microbiota. Gut 67: 1974-1983 (2018)

Winther G, Pyndt Jørgensen BM, Elfving B, Nielsen DS, Kihl P, Lund S, Sørensen DB, Wegener G. Dietary magnesium deficiency alters gut microbiota and leads to depressive-like behaviour. Acta Neuropsychiatr. 27: 168-176 (2015)

Wu G. Intestinal mucosal amino acid catabolism. J. Nutr. 128: 1249-1252 (1998)

Yoshimoto S, Loo TM, Atarashi K, Kanda H, Sato S, Oyadomari S, Iwakura Y, Oshima K, Morita H, Hattori M, Honda K, Ishikawa Y, Hara E, Ohtani N. Obesity-induced gut microbial metabolite promotes liver cancer through senescence secretome. Nature 499: 97-101 (2013)

Yu T, Zhu C, Chen S, Gao L, Lv H, Feng R, Zhu Q, Xu J, Chen Z, Jiang Z. Dietary high zinc oxide modulates the microbiome of ileum and colon in weaned piglets. Front. Microbiol. 8: 825 (2017)

Zackular JP, Moore JL, Jordan AT, Juttukonda LJ, Noto MJ, Nicholson MR, Crew JD, Semler MW, Zhang Y, Ware LB, Washington MK, Chazin WJ, Caprioli RM, Skaar EP. Dietary zinc alters the microbiota and decreases resistance to Clostridium difficile infection. Nat. Med. 22: 1330-1334 (2016)
Ze X, Duncan SH, Louis P, Flint HJ. Ruminococcus bromii is a keystone species for the degradation of resistant starch in the human colon. ISME J. 6: 1535-1543 (2012)

Zhang BB, Liu YM, Hu AL, Xu SF, Fan LD, Cheng ML, Li C, Wei LX, Liu $\mathrm{J} . \mathrm{HgS}$ and Zuotai differ from $\mathrm{HgCl}_{2}$ and methyl mercury in intestinal $\mathrm{Hg}$ absorption, transporter expression and gut microbiome in mice. Toxicol. Appl. Pharmacol. 379: 114165 (2019)

Zhang S, Jin Y, Zeng Z, Liu Z, Fu Z. Subchronic exposure of mice to cadmium perturbs their hepatic energy metabolism and gut microbiome. Chem. Res. Toxicol. 28: 2000-2009 (2015)

Zhu Y, Lin X, Zhao F, Shi X, Li H, Li Y, Zhu W, Xu X, Li C, Zhou G. Meat, dairy and plant proteins alter bacterial composition of rat gut bacteria. Sci. Rep. 5: 15220 (2015)

Zoetendal EG, Raes J, van den Bogert B, Arumugam M, Booijink CCGM, Troost FJ, Bork P, Wels M, de Vos WM, Kleerebezem $M$. The human small intestinal microbiota is driven by rapid uptake and conversion of simple carbohydrates. ISME J. 6: 1415-1426 (2012)

Publisher's Note Springer Nature remains neutral with regard to jurisdictional claims in published maps and institutional affiliations. 